\title{
Nichteinzug der Praxisgebühr in einer Krankenhaus-Notfallambulanz Kein pauschales Zurückbehaltungsrecht des Notfallhonorars durch die KV
}

Kassenärztliche Vereinigungen müssen sich, wenn sie ihr Honorarzurückbehaltungsrecht ausüben, daran orientieren, welche Einzugsquote in der jeweiligen Gruppe der Leistungserbringer leistbar ist. Eine pauschale Kürzung der Abrechnung für den Fall, dass ein Leistungserbringer in einem Quartal in $10 \%$ oder mehr der Behandlungsfälle die Zuzahlung nicht erhoben hat, kann gegebenenfalls eine Ermessensfehlentscheidung darstellen.

Werden Krankenhäuser im ambulanten Bereich tätig, gilt grundsätzlich auch für Krankenhäuser das Erfordernis des Einzugs der Praxisgebühr. Gemäß §28 Abs. 4 des Fünften Sozialgesetzbuchs (SGB V) leisten Versicherte, die das 18. Lebensjahr vollendet haben, je Kalendervierteljahr für jede erste Inanspruchnahme eines an der ambulanten ärztlichen, zahnärztlichen oder psychotherapeutischen Versorgung teilnehmenden Leistungserbringers, wenn keine Überweisung aus demselben Kalendervierteljahr vorliegt, als Zuzahlung die Praxisgebühr an den Leistungserbringer. Die Pflicht für den Patienten, die Praxisgebühr zu entrichten, gilt auch, wenn er ambulante ärztliche Behandlungen in einer Notfallambulanz in Anspruch nimmt, da die Notfallambulanz in diesem Fall an der ambulanten ärztlichen Versorgung teilnehmender Leistungserbringer im Sinne des $\S 28$ Abs. 4 SGB V ist. Die ambulanten Leistungen der Notfallambulanz werden von der Kassenärztlichen Vereinigung über die Honorarbescheide ausgezahlt. Aufgrund bundesmantelvertraglicher Regelungen (§ 18 Abs. 7a BMV-Ä für die Primärkas-

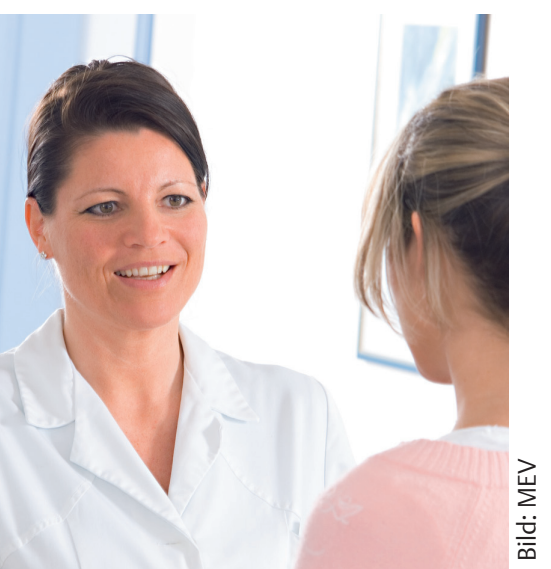

sen bzw. § 21 Abs. 7a EKV-Ä für die Ersatzkassen) kann die Kassenärztliche Vereinigung grundsätzlich die Differenz zwischen einzubehaltender und einbehaltener $\mathrm{Zu}$ zahlungen zurückbehalten, d.h. vom Honorar abziehen, wenn sich aus der Abrechnung ergibt, dass ein Leistungserbringer in einem Quartal in 10\% oder einem höheren Anteil der Behandlungsfälle, in denen die Zuzahlung nach $\S 28$ Abs. 4 SGB V zu erheben ist, die Zuzahlung nicht erhoben hat.

\section{Der Fall}

Im konkreten Fall behielt eine Kassenärztliche Vereinigung Honorarzahlungen in Höhe von ca. 48000,00 Euro für insgesamt 3 Quartale zurück, weil das Krankenhaus die Praxisgebühr in einem erheblichen Umfang nicht eingezogen hatte.

Die Klägerin betreibt als Krankenhausträger unter anderem das betroffene Klinikum, welches in einer Notfallambulanz ambulante vertragsärztliche Leistungen erbringt. Im Rahmen der Honorarbescheide für die betreffenden Quartale machte die Kassenärztliche Vereinigung ein Zurückbehaltungsrecht bezüglich der der Klägerin für erbrachte Notfallleistungen in den streitigen Quartalen zustehenden Honorare in Höhe der nicht einbehaltenen Zuzahlungen geltend und hielt insgesamt 48000,00 Euro zurück. Die Nichteinzugs- bzw. Nichtzahlerquote im Klinikum lag zwischen 59,55\% und $68,80 \%$. Gegen die Zurückbehaltung legte die Klägerin sowohl Widerspruch als auch Klage ein. Dies jedoch ohne Erfolg. Das Landessozialgericht hob auf die Berufung des Krankenhausträgers das Urteil des Sozialgerichts auf und verurteilte die Kassenärztliche Vereinigung, an die Klinik den entsprechenden Betrag nebst Zin- sen ab Rechtshängigkeit zurückzuzahlen. Hiergegen wandte sich nun die Kassenärztliche Vereinigung mit der Revision zum Bundessozialgericht (BSG).

\section{Die Entscheidung}

Das Bundessozialgericht gab der Kassenärztlichen Vereinigung teilweise Recht. So entschieden die Richter, dass das Berufungsurteil insoweit zu ändern sei, als die Beklagte zur Auszahlung des einbehaltenen Betrages nebst Zinsen verurteilt worden sei. Die Kassenärztliche Vereinigung sei vielmehr nur verpflichtet, über die Ausübung des Zurückbehaltungsrechts der Höhe nach unter Beachtung der Rechtsauffassung des Senats erneut zu entscheiden (Urteil des Bundessozialgerichts vom 08.02.2012, B 6 KA 12/11 R). Nach Auffassung des BSG ist das Zurückbehaltungsrecht durch die Kassenärztliche Vereinigung anhand einer den rechtlichen Anforderungen entsprechenden Rechtsgrundlage durchgeführt worden. Auch hätten die tatbestandlichen Voraussetzungen vorgelegen.

Jedoch hätte die Kassenärztliche Vereinigung das ihr zustehende Ermessen fehlerhaft ausgeübt.

Nach Auffassung der Richter gilt die Praxisgebühr auch für die ambulante ärztliche Behandlung, die auf der Grundlage des $\S 76$ Abs. 1 Satz 2 SGB V in Notfällen von „anderen Ärzten“ - vorliegend von der Notfallambulanz eines Krankenhauses - erbracht werden, da diese an der ambulanten ärztlichen Versorgung teilnehmende Leistungserbringer im Sinne des $§ 28$ Abs. 4 SGB V sind. Der Leistungserbringer hat die Praxisgebühr einzubehalten. Sein Vergütungsanspruch gegenüber der Krankenkasse, der Kassenärztlichen Vereinigung oder der Kassenzahnärztlichen Vereinigung verringert sich entsprechend. Die von der Krankenkasse zu entrichtenden Vergütungen verringern sich in Höhe der Summe der von den mit der Kassenärztlichen Vereinigung abrechnenden Leistungserbringern einbehaltenen Zuzahlungen. 
Gläubiger des Anspruchs auf die Praxisgebühr ist damit die Krankenkasse, während die Vertragsärzte (bzw. die Notfallambulanz) lediglich als Einzugs- bzw. Inkassostelle fungieren. Ausfälle gehen somit letztlich zu Lasten der Krankenkasse. Ein Vertragsarzt hat einen Versicherten, der die Praxisgebühr anlässlich einer ärztlichen Behandlung nicht zahlt, lediglich schriftlich zur Nachentrichtung aufzufordern. Bleibt diese Mahnung erfolglos, hat die Kassenärztliche Vereinigung im Auftrag der Krankenkassen die Einziehung der Zuzahlung zu übernehmen. Da die Leistungserbringer an der Einziehung der Praxisgebühr nicht notwendig ein eigenes wirtschaftliches Interesse hätten, haben die Vertragspartner der Bundesmantelverträge vereinbart, dass die Kassenärztlichen Vereinigungen unter bestimmten Voraussetzungen Teile der geschuldeten Vergütung zurückbehalten dürfen. Die Rechtsgrundlage findet sich in $\S 18$ Abs. 7a BMV-Ä (bzw. $§ 21$ Abs. 7a EKV-Ä). Diese Regelungen sind nicht allein für Vertragsärzte verbindlich, sondern gelten auch für andere Leistungserbringer, die vertragsärztliche Leistungen erbringen. Gleichzeitig leitet die Kassenärztliche Vereinigung in Abstimmung mit der zuständigen Krankenkasse ein Schlichtungsverfahren ein. Im Rahmen dieses Schlichtungsverfahrens wird sodann geklärt, inwieweit ein Schadensersatzanspruch der Krankenkassen gegen die Klinik wegen des Nichteinzugs besteht.

Kein undifferenziertes Einbehalten Auch nach Auffassung des BSG lagen grundsätzlich die Voraussetzungen zum Einbehalt vor. In der Notfallambulanz der Klinik wurden die Zuzahlungen unstrittig in weitaus mehr als $10 \%$ der Behandlungsfälle „nicht erhoben“. Mit „erheben“ sei hier das erfolgreiche Einziehen im Sinne der tatsächlichen Realisierung der Praxisgebühr durch Zahlung gemeint. Weitere Tatbestandsvoraussetzungen bestünden nicht. Der Kassenärztlichen Vereinigung stünde bei der Ausübung des Zurückbehaltungsrechts jedoch ein Ermessen zu. Dies ergibt sich aus der Formulierung „kann... zurückbehalten“. Das heißt, es werde ihr die Freiheit eingeräumt, zwischen mehreren als rechtmäßig angesehenen Entscheidungen zu wählen. Das Ermessen der Kassenärztlichen Vereinigung umfasst nach Auffassung der Richter auch die Entscheidung darüber, in welchem Umfang sie von diesem Recht Gebrauch machen. Dies ergäbe sich aus dem Zweck der Regelung. Einer Ermessensausübung bedürfe es bereits deshalb, weil die Rechtsfolge (Zurückbehaltung) sehr weit gefasst sei. Sie gestatte es, die Differenz zwischen einzubehaltender und einbehaltener Zuzahlungen in vollem Umfang zurückzubehalten. Damit erfasse das Zurückbehaltungsrecht auch Fälle, in denen die Nichtzahlung der Praxisgebühr nicht mehr in den Verantwortungsbereich des Leistungserbringers fällt und in denen sich - sofern auch eine Vollstreckung erfolglos bleibt - das Inkassorisiko der Krankenkassen realisiert. Das Gesetz sehe nicht vor, dass die Leistungserbringer die Zuzahlung „erfolgreich” einziehen müssten, sondern ihre Verpflichtung bestünde lediglich darin, die Zuzahlung vom Versicherten einzufordern. Sie ende, wenn der Versicherte trotz gesonderter schriftlicher Aufforderung durch den Leistungserbringer nicht zahle. Die gesetzlichen Regelungen würden belegen, dass auch der Gesetzgeber davon ausginge, dass ein gewisser Teil der Patienten seiner Zahlungspflicht nicht freiwillig nachkäme. Zu diesem Personenkreis gehörten schon diejenigen Patienten, die - selbst bei pflichtgemäßen Einzugsversuchen durch den Leistungserbringer - weder im unmittelbaren $\mathrm{Zu}$ sammenhang mit der Behandlung noch nach schriftlicher Aufforderung durch den Leistungserbringer zu einer Zahlung bereit seien. Ein undifferenziert ausgeübtes Zurückbehaltungsrecht würde dazu führen, dass Honorarzahlungen auch in Fällen zurückgehalten werden dürften, in denen (auch) einen korrekt handelnden Leistungserbringer nach erfolgloser Mahnung keine weiteren Pflichten träfen.

\section{BMV-Regelungen bilden Situation in Notfallambulanzen nicht ab}

Hinzu käme, dass die bundesmantelvertraglichen Regelungen erkennbar auf die Leistungserbringung bei niedergelassenen Vertragsärzten zugeschnitten seien. Sie unterstelle nämlich, dass eine Überschreitung der $10 \%$-Grenze ohne Weiteres ein Zurückbehaltungsrecht rechtfertige, weil ein Nichteinzug in dieser Höhe nur in extremen Ausnahmefällen zu erwarten sei. Dies treffe jedoch für die Leistungserbringung in Notfallambulanzen nicht zu.

Nach Auffassung der Richter bestünde die Gefahr, dass die Kassenärztlichen Vereinigungen sich bei undifferenzierter Aus- übung des Zurückbehaltungsrechts auf die Ausübung des Zurückbehaltungsrechts beschränken und von der ihnen obliegenden Aufgabe der Einleitung eines Verwaltungsverfahrens gegen säumige Zahler absähen. Auch die Kassenärztliche Vereinigung im konkreten Fall hätte es offenbar dabei bewenden lassen, Honorare im Umfang der nicht von der Klinik eingezogenen Zuzahlungen zurückzuhalten und in dieser Höhe einen Schaden bei der Schlichtungsstelle geltend zu machen. Die Anwendung des $\S 18$ Abs. 7a Satz 1 BMV-Ä dürfe jedoch die gesetzlich vorgegebene Aufgaben- und Risikoverteilung nicht unterlaufen.

Eine Kassenärztliche Vereinigung sei daher nicht berechtigt, unter Hinweis auf einen gegebenenfalls insuffizienten Einzug der Praxisgebühr durch den Leistungserbringer die gesetzlich vorgegebenen Maßnahmen zu unterlassen und stattdessen ohne Weiteres die (volle) Differenz als Schaden geltend zu machen. Die Kassenärztliche Vereinigung habe insoweit fehlerhaft gehandelt, weil sie das ihr auch hinsichtlich der Höhe des vorgenommenen Einbehalts zustehende Ermessen weder erkannt noch dieses Ermessen entsprechend dem Zweck der bundesmantelvertraglichen Regelungen ausgeübt habe. Ausgehend von der Zielsetzung des in den Bundesmantelverträgen normierten Zurückbehaltungsrechts als Druckmittel hat die Kassenärztliche Vereinigung im Rahmen ihrer Ermessensentscheidung zu prüfen, ob und in welchem Umfang es in Bezug auf den konkret betroffenen Leistungserbringer dieses Druckes auch tatsächlich bedarf. Die Regelungen berechtigten die Kassenärztliche Vereinigung zur Einbehaltung von Honorar maximal (!) in Höhe der Differenz zwischen den tatsächlich einbehaltenen Zuzahlungen der Versicherten und der vollen Summe der angefallenen $\mathrm{Zu}$ zahlungen. Ob die Kassenärztliche Vereinigung diesen Rahmen ausschöpft, läge in ihrem Ermessen, von dem sie sachgerechten Gebrauch machen müsse. Dies habe zur Folge, dass sie sich je nach Art der Leistungserbringung daran orientieren müsse, welche Ausfallquote tatsächlich unvermeidbar sei.

\section{Vollständiger Einbehalt nur in Extremfällen}

Grundsätzlich ist nach Auffassung der Richter davon auszugehen, dass ein vollständiger Einbehalt des Differenzbetrages 
nur in Extremfällen in Betracht kommt. Dies sei etwa dann der Fall, wenn der Einzug der Zuzahlung grundsätzlich verweigert werde. Dasselbe gelte, wenn die Nichteinzugsquote auch nach Ablauf einer gewissen Eingewöhnungsfrist nach Einführung der „Praxisgebühr” unverändert hoch bliebe, obwohl in einem Schiedsverfahren bestandskräftig festgestellt worden sei, dass die Höhe des Nichteinbehalts ganz wesentlich auf eine schuldhafte Verletzung der dem Leistungserbringer obliegenden Pflichten zurückzuführen ist. Lägen jedoch derartige Fälle nicht vor, verstoße ein über das Maß des Erforderlichen hinausgehender Einbehalt gegen den Grundsatz der Verhältnismäßigkeit.

\section{Konkrete Umstände sind zu prüfen}

Bei der Ermessensausübung sei zunächst in Betracht zu ziehen, dass es eine gewisse Quote von Patienten geben wird, die bei einer Inanspruchnahme des Notfalldienstes die Zahlung der Praxisgebühr generell verweigern und für deren Verhalten die Leistungserbringer keine Verantwortung träfe. Hinzu kämen die Fälle, in denen eine unmittelbare Zahlung an den Leistungserbringer an tatsächlichen Gegebenheiten scheitert. Um entscheiden zu können, ob es bzw. - hiervon nicht zu trennen - in welchem Umfang es der Ausübung des Druckmittels gegenüber einem Leistungserbringer bedürfe, sei daher vorab zu klären, inwieweit die konkreten Umstände der Leistungserbringung Einfluss auf den „erfolgreichen” Einzug der Zuzahlung durch den Leistungserbringer hätten. Da nach Auffassung der Richter signifikante Unterschiede zwischen Arztpraxen und Notfallambulanzen von Krankenhäusern bestünden, sei die $10 \%$-Grenze auf Krankenhausambulanzen nicht einfach übertragbar. So werde - mit einer Ausnahme - in keiner von der Klinik betriebenen Notfallambulanzen eine Zahlungsquote von $50 \%$ erreicht. Ob dazu das Verhalten der Klinik beigetragen habe, werde abschließend im Schlichtungsverfahren zu klären sein.

Schon die Lebenserfahrung spreche allerdings dafür, dass die Nichtzahlerquote in Arztpraxen angesichts einer mehr oder weniger starken Arzt-Patienten-Bindung gering sei, während sie in Notfallambulanzen aus einer Vielzahl von Gründen typischerweise größer sein wird. Eine ArztPatienten-Bindung werde dort in aller Regel fehlen. Es läge weiter nahe, dass eine größere Anzahl von Patienten „unvorbereitet" in die Notfallambulanz komme. Dies gelte zumindest für die „echten” Notfälle. Ein gewisser Teil der Patienten werde also gegebenenfalls keine Zahlungsmittel dabei haben. $\mathrm{Zu}$ berücksichtigen sei auch, dass ein gewisser Teil der Patienten nicht ansprechbar sei und den Patienten auch keine Angehörigen begleiten.

Bei der Prüfung, in welchem Umfang ein Einzug der Zuzahlungen auch bei der Beachtung der gebotenen Sorgfalt überhaupt möglich bzw. realistisch sei, sei zudem zu berücksichtigen, dass nach $\S 18$ Abs. 3 BMV-Ä der Einzug der Praxisgebühr bei „akuter Behandlungsbedürftigkeit” auch nachträglich - das heißt nach Behandlung bzw. Diagnosestellung - erhoben werden kann. Mit „akuter Behandlungsbedürftigkeit" seien Konstellationen gemeint, die eine sofortige, „von Zuzahlungsbemühungen nicht verzögerte", Behandlung erforderlich machten. Akute Behandlungsbedürftigkeit in diesem Sinne dürfte in Notfallambulanzen in deutlich höherem Ausmaß gegeben sein als in den Praxen niedergelassener Ärzte, auch wenn anzunehmen ist, dass die Notfallambulanzen der Krankenhäuser abends und am Wochenende auch von zahlreichen Patienten aufgesucht würden, die die Voraussetzungen für einen „Notfall” nicht erfüllen. Zwar habe die Kassenärztliche Vereinigung zutreffend darauf hingewiesen, dass auch in Fällen akuter Behandlungsbedürftigkeit die Möglichkeit zur Einziehung nachträglich bestehe. Jedoch dürfte sich der Druck auf den Patienten, die Praxisgebühr zu entrichten, nach Durchführung der Behandlung deutlich vermindert haben. Dies stütze die Annahme, dass die Zahl der Nichtzahler in Notfallambulanzen naturgemäß deutlich höher ist als in Arztpraxen.

\section{Ausfallquoten vergleichbarer Einrichtungen berücksichtigen}

Es läge nach alledem nahe, dass die $10 \%$-Grenze in Notfallambulanzen regelhaft überschritten werde, ohne dass dies den Leistungserbringern angelastet werden könne. Zumindest bis ein erstes Schlichtungsverfahren bestandskräftig abgeschlossen sei, müsse sich die Höhe der Ausfallquote, auf die eine Kassenärztliche Vereinigung ihr Zurückbehaltungsrecht bei pflichtgemäßer Ermessensausübung erstreckt, daher an dem orientieren, was in Notfallambulanzen von Krankenhäusern leistbar sei. Die Kassenärztliche Ver- einigung müsse den Umfang der typischerweise unvermeidbaren Nichtzahlungen nicht exakt ermitteln, aber doch der Größenordnung nach berücksichtigen. Sie habe daher vor der Zurückbehaltung einer bestimmten Summe die Frage zu klären, welche Ausfall- bzw. Nichteinzahlungsquoten sich in vergleichbaren Einrichtungen ergeben. Es sei daher sachgerecht, wenn die Beklagte ermittle, wie hoch die Ausfallquote in vertragsärztlichen Notfalleinrichtungen sei, also bei Patienten, die einen ihnen fremden Arzt im Quartal nur wegen einer Notfallversorgung aufgesucht hätten. Aussagekräftig könnten auch die Werte von anderen Klinikträgern im Bereich der Beklagten sowie aus anderen Kassenärztlichen Vereinigungs-Bezirken sein. Wenn diese Ermittlungen ergäben, dass die Nichteinzugsquote auch bei sachgerechter Organisation generell im Notfalldienst wegen der Besonderheiten der Patientenklientel und der Art der Behandlungen Werte von z.B. $30 \%$ oder $35 \%$ erreiche, dürfe die Beklagte (jedenfalls vor bestandskräftigem Abschluss des ersten Schlichtungsverfahrens) nicht einfach unterstellen, die Klägerin hätte eine Ausfallquote von mehr als $10 \%$ verhindern können und müssen.

\section{Fazit}

Das Urteil stärkt zwar vordergründig die Rechte der Krankenhausträger, pauschale Kürzungen der Kassenärztlichen Vereinigungen in voller Höhe nicht hinnehmen zu müssen. Es ist jedoch kein Freibrief, auf den Einzug der Praxisgebühr zu verzichten. Die Verpflichtung, die Praxisgebühr soweit wie möglich einzuziehen, besteht derzeit noch. Spätestens im Schlichtungsverfahren wird es erforderlich sein, dezidiert darzulegen, warum eine gewisse Ausfall- bzw. Nichteinzugsquote vorlag und darzulegen, dass der Ausfall nicht auf Fehlern oder Desinteresse der Klinik beruht.

Das Urteil gibt jedoch zahlreiche Anhaltspunkte im Hinblick auf die Höhe der zurückzubehaltenden Beträge, die sich so auch im Bescheid wiederfinden sollten.

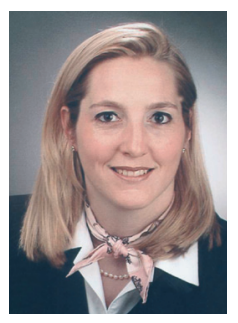

Korrespondenz Dr. iur. Isabel Häser Rechtsanwältin Ehlers, Ehlers und Partner Widenmayerstr. 29 80538 München 